Тихонова А.C.*, Котельникова А.В., Кукшина А.A.

Возможности объективизации и разработка критериев оценки динамики показателей стандартных нейропсихологических методик

Московский научно-практический центр медицинской реабилитации, восстановительной и спортивной медицины

Департамента здравоохранения города Москвы, ул. Земляной Вал, 53, стр.1, Москва, 105120, Россия. seyli1992@ list.ru*

Статья поступила 05 ноября 2020; принята 10 декабря 2020; опубликована 31 декабря 2020

Аннотация. Активное развитие комплексной нейрореабилитации и переформатирование клинической составляющей в рамках функционирования мультидисциплинарной реабилитационной бригады в методическом плане обосновывает необходимость поиска новых способов динамической оценки состояния высших психических функций, сочетающих в себе объективизацию измерений, индивидуальный подход и достаточную степень научного обобщения. Цель работы: верификация предположения о возможности применения кластерного анализа как метода объективизации оценки высших психических функций в процессе медикопсихологической реабилитации. Материалы и методы: участники исследования 93 пациента (перенесшие острые нарушения мозгового кровообращения (ОНМК) - n=57; пациенты с дегенеративно-дистрофическими заболеваниями крупных суставов и позвоночника $-\mathrm{n}=36$ ), находившиеся на стационарном этапе медицинской реабилитации, выполнявшие задания по методикам «Таблицы Шульте» и «Заучивание 10 слов» А.Р. Лурия. Полученные результаты обрабатывались методами математической статистики с использованием кластерного анализа по методу К-средних. Результаты исследования и их обсуждение: использование процедуры кластерного анализа по методу К-средних на примере исследования характеристик памяти и внимания с учетом нозологических различий и их динамики в ходе психокоррекционного воздействия позволило при анализе результатов применения стандартных нейропсихологических методик дискриминировать обследованную выборку в количественных градациях «низкого / среднего / высокого» уровня выраженности изучаемых признаков с учетом многофакторной структуры эмпирических данных и решить вопрос объективизации специфических локальных данных, нивелируя проблему поиска единых нормативов. Заключение: в рамках исследования был разработан новый подход к обработке данных нейропсихологической диагностики посредством применения при анализе результатов процедуры кластерного анализа по методу К-средних, позволяющий объективизировать оценку высших психических функций в процессе медико- 
социальной реабилитации и интегрировать полученные данные в общенаучный контекст.

Ключевые слова: нейропсихологическое исследование; кластерный анализ; нейрореабилитация; инсульт; виртуальная реальность.

Информация для цитирования: Тихонова А.С., Котельникова А.В., Кукшина А.А. Возможности объективизации и разработка критериев оценки динамики показателей стандартных нейропсихологических методик // Научный результат. Педагогика и психология образования. 2020. Т.6. №4. C. 81-95. DOI: 10.18413/23138971-2020-6-4-0-7.

\title{
A.S. Tihonova*, A.V. Kotel'nikova, A.A. Kukshina Possibilities of objectification and development of criteria
for the analysis of the indicators used by the standard
neuropsychological methodologies
}

Moscow Centre for Research and Practice in Medical Rehabilitation, Restorative and Sports Medicine, Moscow Healthcare Department, 53 bld. 1, Zemlyanoy Val Str., Moscow, 105120, Russia seyli1992@list.ru*

Received on November 05, 2020; accepted on December 10, 2020; published on December 31, 2020

\begin{abstract}
The active development of complex neurorehabilitation and reformatting of the clinical component within the framework of the multidisciplinary rehabilitation team operation methodically justifies the necessity of discovering new ways to dynamically assess the higher mental functions, which would combine objectification of measurements, an individual approach and a sufficient degree of scientific generalisation. Aim of the study: to verify the hypothesis of suitability of application of the cluster analysis, as a higher mental functions assessment objectification method, in medicalpsychological rehabilitation. Methodology and methods: the study included 93 participants (of whom $57(n=57)$ had suffered an acute cerebrovascular event (ACE) and 36 $(n=36)$ were patients with degenerative-dystrophic diseases of large joints and the spine), all of whom were in the in-patient stage of their medical rehabilitation. The patients were assigned tasks according to the Schulte Table and the A.R. Luria's 10 Words methods. The results were processed using the mathematical statistical methods of Kmean clustering. Research Results and Discussion: application of K-mean clustering to the analysis of the memory and attention, taking into account nosological differencies and their dynamics during psychocorrection, allowed to discriminate the studied sample against "low/moderate/high" scale of intensity of the studied characteristics while considering the multifactorial structure of the empirical data, and to objectify specific local data thus mitigating the issue of unified standards during the analysis of the standard neuropsychological methods. Concluions: a new approach utilising K-mean clustering in processing neuropsychological diagnostic data was developed during the study. The approach allowed for the objectification of the higher mental functions to be carried out during the process of socio-medical rehabilitation and for the resulting data to be integrated within the existing general scientific context.
\end{abstract}


Keywords: neuropsychological study; clustering; neurorehabilitation; stroke; virtual reality.

Information for citation: Tihonova A.S., Kotel'nikova A.V., Kukshina A.A. (2020), "Possibilities of objectification and development of criteria for the analysis of the indicators used by the standard neuropsychological methodologies", Research Result. Pedagogy and Psychology of Education, 6 (4), 81-95, DOI: 10.18413/2313-8971-2020-6-4$0-7$.

Введение (Introduction). Активное развитие комплексной нейрореабилитации и переформатирование клинической составляющей в рамках функционирования мультидисциплинарной реабилитационной бригады в методическом плане не только ставит вопрос о выработке междисциплинарного терминологического аппарата, понятного всем участникам команды, но и делает актуальным поиск эргономичных инструментальных способов динамической оценки состояния пациента в процессе реабилитации, сочетающих в себе стремление к объективизации измерений, персонифицированный клинический подход и достаточную степень научного обобщения.

Применительно к обозначенной проблеме континуум методов нейропсихологического исследования помещает специалиста в ситуацию выбора между качественным феноменологическим подходом к анализу результатов - с одной стороны: и психометрическим, основанном на едином принципе стандартизации процедур проведения тестов, - с другой (Butler M., Retzlaff P., Vanderploeg R., 1991; Lezak M.D., Howieson D.B., Bigler E.D., Tranel D., 2012). Значимыми достоинствами последнего являются доказанная валидность используемых инструментов, разработанность возрастных, социокультурных и иных нормативов, позволяющих получить данные об испытуемом в сравнении со среднестатистической нормой соответствующей выборки (Астаева, Солянникова, 2018; Ахутина, Меликян, 2012; Беребин, Астаева, 2008), объективизация и воспроизводимость результатов, а также подтверждение или опровержение их различными авторами (Ахутина, Меликян, 2012; Вассерман, Щелкова, 2003; Логвино- ва, 2010; Микадзе, 2008). К несомненным достоинствам качественного подхода следует отнести возможность выявления структуры и механизмов нарушения, что является значимым критерием при составлении программы коррекции высших психических функций (ВПФ), вариативность и гибкость в использовании тестов в зависимости от текущего состояния ВПФ, что особенно эффективно в диагностике индивидуального случая.

Одним из распространенных вариантов психометрического подхода является развивающаяся в последние годы практика использования скрининговых методик, существенным образом упрощающих работу исследователя, сокращающих время проведения обследования, позволяющих получать стандартизированные результаты, поддающиеся количественной оценке (Рассказова, Ковязина, Варако, 2016; Folstein M.F., Folstein S.E., McHugh P.R., 1975; Golden C.J., 1981; Lerner A.J., 2013; Nasreddine Z.S., Phillips N.A., Bédirian V., Charbonneau S., WhiteheadV., CollinI., Cummings J.L., Chertkow H., 2005). Данный подход имеет свои ограничения, среди которых выделяются недостаточная глубина анализа, ригидность воспроизведения, слабые возможности оценки некоторых базовых функций (память, внимание, речь) (Астаева, 2008). Результаты обследования зачастую сводятся к простому перечислению симптомов нарушений при недостаточности отражения всей диагностической значимости качественной специфики выполнения теста, что затрудняет разработку индивидуализированных стратегий вмешательства и коррекции (Ахутина, Меликян, 2012; Беребин, Астаева, 2008; Глозман, 1999; Микадзе, 2008), а при использо- 
вании специалистами непсихологического профиля может приводить к нарушению процедуры диагностики и поверхностному анализу результатов (Рассказова, Ковязина, Варако, 2016).

Вышеизложенное позволяет переформулировать актуальную проблему современной нейропсихологии в направлении создания методических разработок и диагностических инструментов, совмещающих в себе достоинства качественного и количественного подходов (Ахутина, Матвеева, Романова, 2012; Ахутина, Меликян, 2012; Беребин, Астаева, 2008; Foreman M.D., Fletcher K., Mion L.C., Simon L., 1996; Byrne В., 2016). Необходимо отметить, что различными авторами обозначались ранее возможные варианты такого синтеза, основанные на разработке балльной системы оценки изучаемых параметров для методик, изначально имеющих феноменологический характер интерпретации.

Вассерман Л.И. с соавторами предлагает использовать четырех-балльную систему оценки каждого предлагаемого задания с последующим построением профиля успешности выполнения отдельных проб методики, отражающих особенности и степень выраженности нарушений отдельных высших психических функций (ВПФ) и их взаимосвязи в виде нейропсихологических синдромов (Вассерман, Дорофеева, Меер$\mathrm{coH}, 1997)$.

В работах Ахутиной Т.В. представлен метод количественной обработки результатов обследования на основе усреднения данных функционально близких параметров выполнения проб, для чего были разработаны три индекса, в которые вошли наиболее однозначно интерпретируемые параметры выполнения различных проб (Ахутина, Каширская, 2000).

Еще одна система количественной оценки проб разработана Глозман Ж.М., подход которой основан на факторном качественном анализе. Система позволяет определить наличие симптомов и их количественную оценку, выявить нейропсихологический паттерн, а также предоставляет воз- можность провести группировку качественных параметров на основании их общей отнесенности к работе определенных структур мозга с целью сравнения с нормативами или для оценки динамики состояния (Глозман, 2009).

В работах Микадзе Ю.В. использовались пробы из общего нейропсихологического обследования и оценивалась частотность проявления соответствующей симптоматики. Для оценки структуры выявленного дефицита проводился метасиндромный анализ, заключающийся в описании синдромокомплекса, характеризующего выявленные расстройства различных когнитивных функций с учетом частотности их проявления в обследованной группе больных, а также факторного состава этого синдромокомплекса (указывающего на характеристики функциональной дефицитарности различных зон мозга) (Микадзе, Титкова, Ширшов, Гуща, 2013: 5).

Однако использование методов количественной обработки информации встречает критику в плане обеспечения единственности приписывания числовых значений изучаемым феноменам в полном соответствии с эмпирически сложившимися отношениями в проявлениях каждого феномена и отношениями между феноменами в целом (Беребин, Астаева, 2008: 26). Также должна быть обеспечена равноинтервальность прироста «патологии» на шкале баллов. Так, в работе Беребина М.А. и Астаевой А.В. обсуждается, что результаты использующихся в описываемых балльных системах приемов усреднения интервальных данных затруднительно использовать в параметрической статистике, то есть представляется невозможным получение статистических норм нейропсихологических методик на основе традиционного Z-преобразования результатов исследования в одну их известных психометрических шкал. В качестве решения проблемы авторы предлагают рассмотрение данных нейропсихологического исследования не в рамках статистических норм, полученных путем преобразования нормально распределенных результатов испытуемых, а 
в системе процентильных норм (Беребин, Астаева, 2008: 27).

Другой значимой проблемой в диагностике ВПФ является разработка статистических норм для сравнения результатов выборки, отражающих критерии пола, возраста и социально-культурных особенностей (Карвасарский, 2004; Golden, Espe-Pfeife, Wachesler-Felder, 2002).

Наиболее широко вопрос определения норм освещается в области детской нейропсихологии. Проблема разработки нормативных возрастных данных в детском возрасте связана с отсутствием четкого критерия, характеризующего континуум представлений между патологическими и нормальными симптомами (Микадзе, 2008), а также с вопросом определения нейропсихологических коррелятов индивидуальных различий (Астаева, 2008; Астаева, Солянникова, 2018; Цветкова, 2001).

Зачастую при интерпретации результатов авторы ссылаются на нормативные показатели, указанные в достаточно давних работах, в том числе на отмеченные позиции специалистов, которые вводили эти методики в научную деятельность. При этом известно, что культурно обусловленный процесс развития ВПФ может реагировать на общественные изменения, затрагивающие различные сферы социального функционирования, в связи, с чем учеными поднимается вопрос о необходимости верификации нормативных данных, определенных ранее (Султанова, Иванова, 2017; Golden, Espe-Pfeife, Wachesler-Felder, 2002).

Обозначенные методические сложности наиболее ярко прослеживаются на примере использования таких популярных диагностических методик, как «Таблицы Шульте» и «Запоминание 10 слов» А.Р. Лурия, широко используемых как в рамках диагностики индивидуального случая в клинике, так и в научных исследованиях.

Определение нормативных показателей при выполнении методики «Таблицы Шульте» осложняется тем, что в литературе разнятся данные о характеристиках стимульного материала и нормативного време- ни. Так, предлагается использование таблиц формата 50x50 см с затрачиванием на выполнение поиска всех чисел таблицы порядка 30-50 секунд, чаще всего 40-42 секунды (Рубинштейн, 1999), такое же нормативное время - 40-50 секунд - приводится в работе Менделевича В.Д. (Менделевич, 2008). Другие авторы предлагают использовать таблицы размера 60х60, без указания нормативного времени (Блейхер, Крук, 1986), либо таблицы формата А-4 (Балашова, Ковязина, 2016) с нормой выполнения 30-40 секунд (Харисова, Чебакова, 2018).

Данные о подходах к дифференциации нормативных показателей в зависимости от возраста взрослых испытуемых имеют определенные противоречия и в литературе освещены не столь широко. При использовании методики «Запоминание 10 слов» Рубинштейн С.Я. указывает, что кривая запоминания взрослых здоровых людей и детей школьного возраста не имеет принципиальных отличий (Рубинштейн, 1999). Рядом авторов описаны сходные нормативные данные, выявленные в исследованиях на большом количестве здоровых испытуемых (Блейхер, Крук, 1986; Лурия, 1962; Рубинштейн, 1999), при этом отсутствует указание на достаточный объем отсроченного воспроизведения. В работах Глозман Ж.М. встречаются данные о различных нормативах выполнения методик «Запоминание 10 слов», включая показатели отсроченного воспроизведения, и «Таблицы Шульте» для взрослых людей в зависимости от возраста и уровня образования (Глозман, 2012).

Преодоление описанных затруднений видится в принципиально ином подходе к анализу результатов традиционно используемых методик диагностики индивидуального случая. Современные методы математико-статистической обработки, в частности, многомерный кластерный анализ (Крамер, 2007; Наследов, 2007; Петри, 2015; Ramachandran K.M., Tsokos C.P., 2009; Lang T.A., Secic M., 2006), предоставляют исследователю возможность эргономичных методических решений, соединяющих достоинства индивидуального подхода с возможностью 
интегрировать полученную информацию в общенаучный контекст.

Таким образом, цель настоящего исследования состояла в верификации предположения о возможности применения кластерного анализа, как метода объективизации оценки высших психических функций в процессе медико-психологической реабилитации.

\section{Материал и методы (Methodology} and methods). Всего в исследование было включено 93 пациента, находящихся на стационарном этапе медицинской реабилитации по поводу двигательных последствий острого нарушения мозгового кровообращения (ОНМК) $-\mathrm{n}=57$, среди них было 32 $(56,1 \%)$ женщины и 25 (43,9\%) мужчин в возрасте $60,5 \pm 12,4$ лет; давность инсульта до 6 месяцев; и двигательных нарушений на фоне хронически текущих дегенеративнодистрофических заболеваний (ДДЗ) крупных суставов и позвоночника $(\mathrm{n}=36)-24$ $(66,7 \%)$ женщин и $12(33,3 \%)$ мужчин в воз-

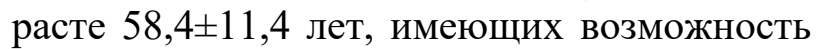
самостоятельно перемещаться в пределах стационара и обслуживать себя. Все пациенты, согласно алгоритму, нуждались в проведении психокоррекционных мероприятий в связи с имеющимися признаками тревоги и депрессии в текущем эмоциональном состоянии (Кукшина, Котельникова, Турова, Рассулова, 2017). После включения пациентов в исследование была произведена рандомизация методом случайных чисел в две группы: основную $(\mathrm{n}=56)$ и контрольную $(n=37)$. В обеих группах реализовывалась программа медицинской реабилитации в соответствии с нозологией двигательных нарушений, при этом психологическое сопровождение было организовано следующим образом: пациенты, вошедшие в контрольную группу, получали стандартный формат вмешательств в рамках пациентцентрированного подхода («Школа пациента», релаксационные техники, психологическое консультирование); в индивидуальный реабилитационный план пациентов основной группы помимо этого были включены также релаксационные процедуры с исполь- зованием программно-аппаратного комплекса бинауральных акустических биений ПРАК ${ }^{1}$ в количестве 8-10 процедур на каждого пациента (1 процедура ежедневно). Бинауральные ритмы (слуховой феномен, возникающий при одновременной подаче в правое и левое ухо звуковых тонов, различающихся на несколько герц, и заключающийся в возникновении биений, равных по частоте разнице между тонами), совпадающие с дельта- и тета-диапазонами электроэнцефалограммы, связываются с состояниями расслабления, медитации и творчества и применяются в качестве седативного средства, помогающего уснуть (Шумов, Арсеньев, Свешников, Дорохов, 2017). Выбор вариантов психокоррекциии и вероятная положительная динамика когнитивного статуса испытуемых в данном случае обусловлены тем, что в основе формирования как тревожно-депрессивного симптомокомплекса, так и когнитивного снижения, лежат тождественные нейромедиаторные процессы, что и позволяет ожидать улучшения когнитивных функций на фоне стабилизации психоэмоционального состояния.

Внимание оценивалось с помощью методики «Таблицы Шульте», позволяющей измерить объем внимания, скорость протекания психических процессов, уровень работоспособности (Ахметзянова, 2013), характеристики памяти - по данным методики «Заучивание 10 слов» Лурия А.Р. (Балашова, Ковязина, 2016). Исследуемые параметры, подлежащие динамическому наблюдению, были измерены дважды: до и после экспериментального воздействия, то есть в начале пребывания пациентов в стационаре и непосредственно перед выпиской.

Математико-статистическая обработка и анализ данных производилась в про-

\footnotetext{
${ }^{1}$ Уникальный номер реестровой записи 39758 , Регистрационный номер медицинского изделия № ФСР 2012/14162, дата государственной регистрации медицинского изделия 06.12.2019, Комплекс аппаратнопрограммный для коррекции психосоматического состояния человека с помощью запрограммированных резонансно-акустических колебаний сигналов ЭКГ и/или ЭЭГ здорового человека Кап КПС-«ЭКРАН» по ТУ 9444722-05834388-2002.
} 
граммном пакете «Statistica 10.0» и включала в себя анализ данных описательной статистики, анализ достоверности сдвига для связанных выборок по Т-критерию Вилкоксона, анализ значимости различий в уровне выраженности количественного признака для несвязанных групп по U-критерию Манна-Уитни, анализ различий в пропорциональной представленности бинарного признака в исследовательских группах по критериям Фишера, кластерный анализ по методу К-средних. Выявленные связи и различия считались достоверными при достижении уровня статистической значимости $\mathrm{p} \leq 0,05$.

Результаты и их обсуждение (Research Results and Discussion). Исследование эффективности психокоррекционного воздействия в отношении когнитивного статуса пациентов с нарушением двигательных функций в процессе медицинской реабилитации осуществлялось в два этапа.

На первом этапе работы производилась оценка состояния внимания и памяти обследованных пациентов $(\mathrm{n}=93)$ до начала реабилитационных мероприятий с учетом нозологических различий. Общая совокупность полученных данных с учетом нозологии двигательных нарушений была подвергнута процедуре кластерного анализа по методу К-средних. Предложенное кластерное решение состояло в выделении в каждой нозологии трех кластеров, соотносящихся с незначительными, умеренными и выраженными нарушениями внимания и памяти.

По результатам применения методики «Таблицы Шульте» в кластер с незначительными нарушениями внимания в группе больных с ОНМК вошло 33 (58,9\%) человека, в группе больных с ДДЗ - 14 (37,8\%) человек; с умеренными нарушениями - 11 $(19,6 \%)$ и $16(43,2 \%)$ человек, с выраженными нарушениями - 12 (21,5\%) и $7(19,0 \%)$ человек соответственно. Использование статистического критерия Фишера для анализа пропорциональной представленности степени выявленных нарушений внимания в нозологических группах позволило зафиксировать, что нарушения внимания выра- женной степени фиксируются приблизительно в 20,0\% случаев в обеих нозологиях $(p=0,49)$, умеренные нарушения чаще встречаются в группе пациентов с ДД3 $(\mathrm{p}=0,01)$, незначительные - в группе пациентов с OНMК $(p=0,04)$. Описанный результат свидетельствовал о необходимости проведения дифференцированного подхода к анализу с учетом нозологической принадлежности и степени выявленных нарушений ВПФ.

На рис. 1 представлена графическая иллюстрация, отражающая динамику выполнения проб по «Таблицам Шульте» в нозологических группах: по вертикальной оси - время работы с таблицей в секундах, по горизонтальной - номера таблиц.

Как видно на рис. 1, нозологическая картина нарушений внимания у обследованных пациентов различна, причем особенно это касается тех случаев, когда нарушения носят умеренный или выраженный характер. Пациенты с перенесенным ОНМК демонстрируют гораздо меньший объем внимания и продуктивность работоспособности, низкую скорость выполнения проб. Относительно ровный, приближенный к прямой линии, характер кривой для всех трех групп пациентов с ОНМК свидетельствовал о присутствующей ригидности психических процессов, в противоположность пациентам с ДД3, где во всех группах с различной степенью нарушения внимания присутствовали выраженные колебания работоспособности, отражаемые ломаным характером линии. Врабатываемость и увеличение скорости выполнения проб из всех обследованных пациентов отмечалось только в группе с незначительными нарушениями внимания в ДД3-нозологии, при этом в группах с умеренными и выраженными нарушениями внимания в этой же нозологии отмечалась повышенная истощаемость психических процессов, что, вероятнее всего, могло быть связано с вторичным (в противоположность первичному у пациентов с ОНМК) нарушением кровоснабжения головного мозга. В целом значения, близкие к условно-нормативным, отмечались в груп- 
пах с незначительными нарушениями в обе-

OHMK их нозологиях (Рубинштейн, 1999).

ДДЗ

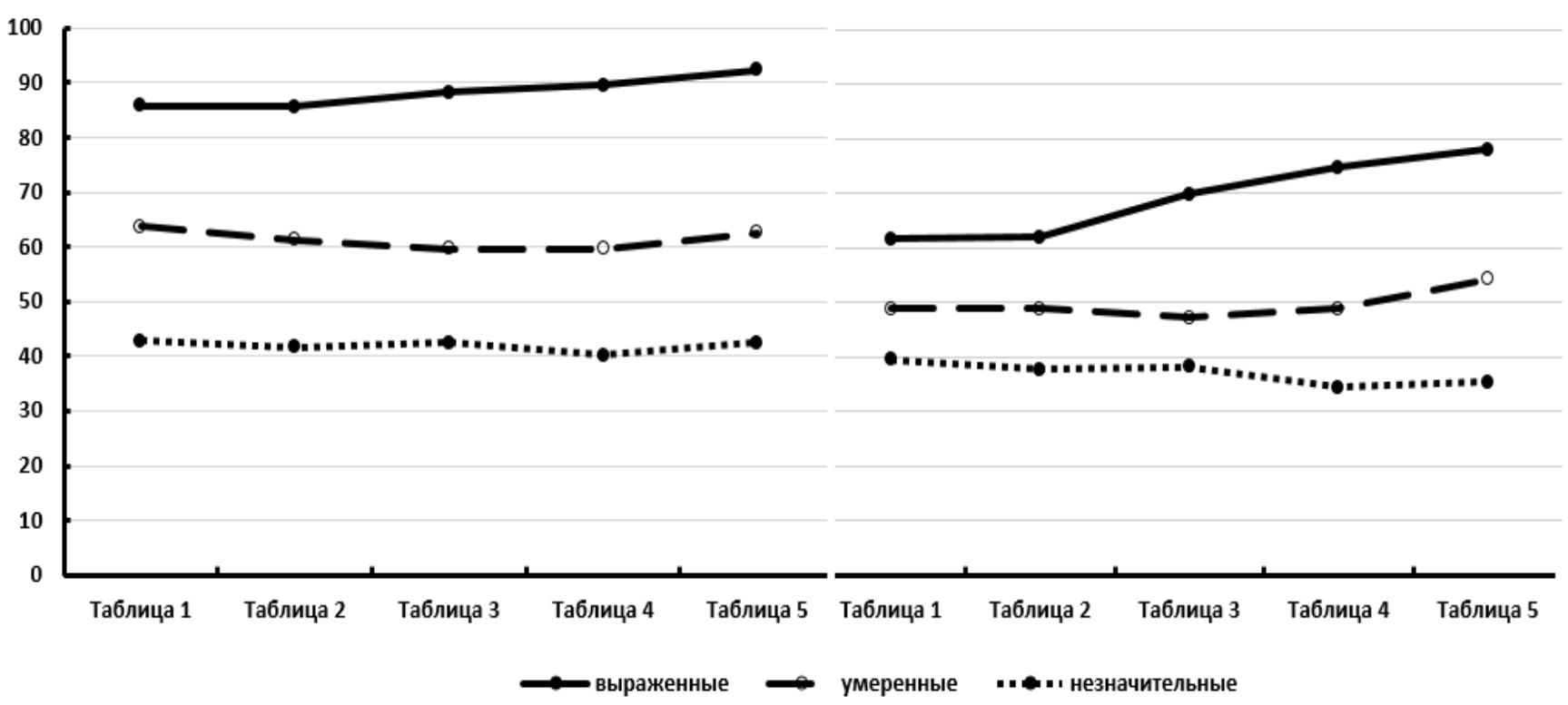

Рис.1 Время выполнения проб по «Таблицам Шульте» в группах

Fig.1 Sample execution time for "Schulte Tables" in groups

Таким образом, внимание в обследованных группах различается в зависимости от нозологии двигательных нарушений: невысокий объем внимания, низкая продуктивность работоспособности и ригидность протекания психических процессов характерны для пациентов с ОНМК, истощаемость и лабильность - для пациентов с ДДЗ, при этом нарушения внимания выраженной степени фиксируются приблизительно в 20,0\% случаев в обеих нозологиях, умеренные нарушения чаще встречаются в группе пациентов с ДД3, незначительные - в группе пациентов с ОНМК.

Предложенное кластерное решение для оценки памяти на основании данных методики «Заучивание 10 слов» также содержало по три кластера в каждой нозологии в зависимости от степени выявленных нарушений памяти: слабые / средние / сильные. В кластер со слабыми нарушениями в группе больных с ОНМК вошло 16 (28,6\%) человек, в группе больных с ДДЗ 9 (24,4\%) человек; со средними нарушениями - $29(51,8 \%)$ и $14(37,8 \%)$ человек, с сильными нарушениями - $11(19,6 \%)$ и 14 (37,8\%) человек, соответственно. Использование статистического критерия Фишера для анализа пропорциональной представленности степени выявленных нарушений памяти в нозологических группах позволило зафиксировать достоверные различия только по частоте представленности сильных нарушений памяти, чаще встречающихся у пациентов с ДДЗ крупных суставов и позвоночника.

Графическая иллюстрация, отражающая динамику выполнения проб по методике «Запоминание 10 слов» в нозологических группах, представлена на рис. 2, где по вертикальной оси отображено количество воспроизведенных слов, по горизонтальной номера попыток. 
онмк

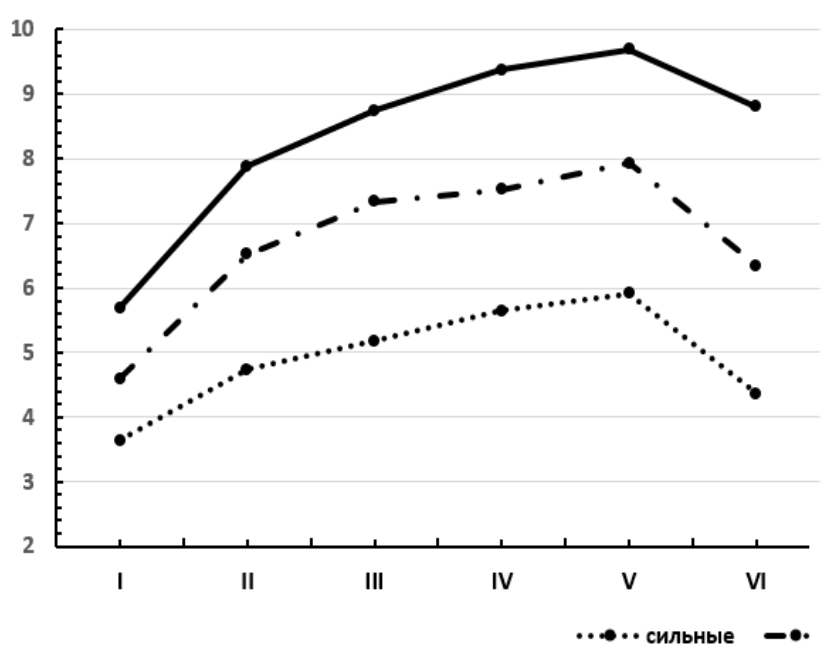

ддз

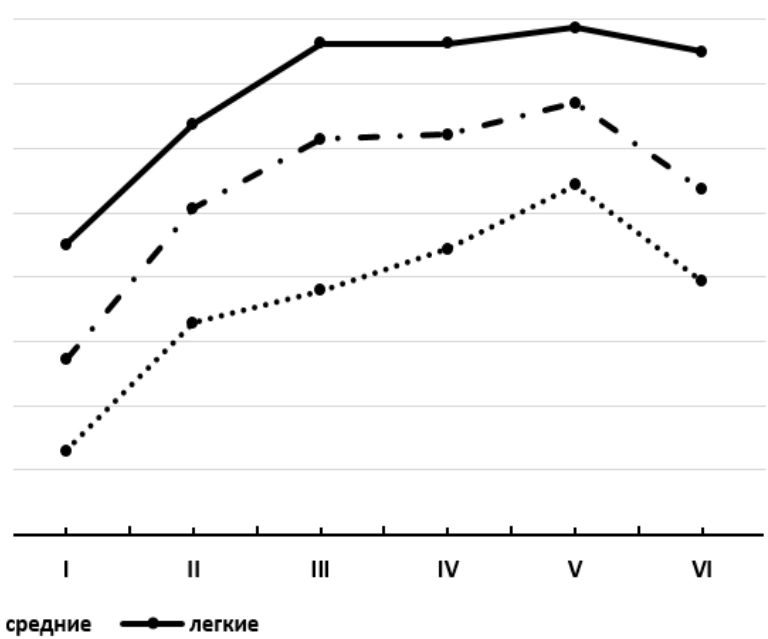

Рис. 2 Динамика выполнения проб по методике «Заучивание 10 слов» в группах Fig. 2 Dynamics of performing test susing the "Memorizing 10 words" methodin groups

Сопоставление графиков, представленных на рис. 2, позволило сделать вывод об относительно большей степени сохранности слухоречевой памяти у пациентов с ДДЗ по сравнению с обследованной группой пациентов с ОНМК. Объем кратковременной памяти, который выявлялся по данным первой попытки воспроизведения, у больных, перенесших инсульт, в двух подгруппах из трех (со средними и с сильными нарушениями) был меньше нормативных значений $(7 \pm 2)$, в группе с ДД3 - в двух кластерах из трех (со средними и со слабыми нарушениями) соответствовал нормативным значениям.

Показательна также графически фиксируемая разница, отражающая влияние гетерогенной интерференции на отсроченное запоминание в нозологических группах: слухо-речевые следы памяти у пациентов из группы ДДЗ значительно более устойчивы, нежели у пациентов с ОНМК, то есть введение интерференции у них не оказывает существенного влияния на объем слухоречевой памяти - в отсроченном периоде эти пациенты чаще всего воспроизводят нормативное количество слов-стимулов (более 5).

Таким образом, предварительное обследование состояния когнитивных функ- ций выявило нозологические различия, а именно: для пациентов с ОНМК характерен невысокий объем внимания и кратковременной памяти, низкая продуктивность работоспособности, ригидность протекания психических процессов.

Второй этап работы был посвящен анализу данных, отражающих динамику когнитивных функций пациентов в результате психологического сопровождения.

Анализ достоверности изменений в уровне выраженности характеристик внимания, по данным методики «Таблицы Шульте», проводился с учетом выделенных на первом этапе исследования кластеров пациентов обеих нозологий двигательных нарушений, в соответствии с выявленными незначительными / умеренными / выраженными нарушениями внимания. Анализ пропорциональной представленности пациентов, вошедших в соответствующие кластеры после рандомизации в основную (ПРАК) и контрольную группу, по критерию Фишера достоверных различий не выявил $(\mathrm{p}>0,05)$. В основную группу вошли 12 пациентов с выраженными нарушениями внимания $(21,4 \%), 19(33,9 \%)$ - с умеренными, 25 $(44,7 \%)$ - с легкими; соответствующие показатели в контрольной группе составили 6 $(16,2 \%), 8(21,6 \%)$ и $23(62,2 \%)$. Нозологи- 
ческие различия в характеристиках внимания, выявленные на первом этапе исследования, при дальнейшем анализе также учитывались.

Достаточный для подтверждения достоверности произошедших изменений в процессе медицинской реабилитации уровень статистической значимости Т-критерия Вилкоксона $(\mathrm{p} \leq 0,05)$, по итогам выполнения методики «Таблицы Шульте» до и после психокоррекции в основной и контрольной группах, был зафиксирован только дважды в основной группе: снизилось время выполнения таблицы 2, то есть несколько повысилась врабатываемость $(\mathrm{p}=0,01)$ у пациентов c выраженными нарушениями внимания, перенесших ОНМК, при этом у пациентов с незначительными нарушениями внимания повысилась утомляемость $(\mathrm{p}=0,003)$, что отразилось в увеличении времени выполнения таблицы 4. Несмотря на то, что в контрольной группе достоверное изменение показателей не было зафиксировано ни в одном случае, разнородность описанного результата не позволяет сделать однозначный вывод о целесообразности применения программно-аппаратного комплекса ПРАК для психологической коррекции внимания у обследованной когорты пациентов.

Вопрос о динамике показателей, характеризующих память обследованных пациентов, рассматривался с учетом выявленных на первом этапе исследования нозологических различий. Использовался Ткритерий Вилкоксона. Статистически достоверные изменения $(\mathrm{p} \leq 0,05)$ в выполнении проб методики «Заучивание 10 слов», отражающих динамику психических процессов слухо-речевой памяти у обследованных пациентов, были зафиксированы в незначительном количестве случаев, при этом в подавляющем большинстве речь шла даже о некотором ухудшении. Так, достоверное снижение объема следовой памяти произошло во всех группах пациентов (со слабыми / средними / сильными нарушениями памяти), перенесших ОНМК, в программы психокоррекционной реабилитации которых был включен ПРАК, а также в контрольной группе у пациентов с выявленными ранее слабыми нарушениями памяти на фоне ОНМК. Статистически значимое улучшение было зафиксировано только в одном случае, для пациентов с сильными нарушениями памяти из нозологической группы «ДДЗ крупных суставов и позвоночника»: в результате проведения психокоррекционных мероприятий с использованием ПРАК у них было зафиксировано возрастание объема воспроизведения в начале обучения, в процессе первой попытки.

Таким образом, анализ значимости различий в характеристиках когнитивного статуса пациентов с нарушением двигательных функций в результате ОНМК или на фоне ДДЗ крупных суставов и позвоночника в процессе психологического сопровождения в процессе медицинской реабилитации, позволяет сделать вывод об индифферентном ответе психических процессов внимания и слухо-речевой памяти в ответ на введение в план психокоррекционных мероприятий занятий с программно-аппаратным комплексом ПРАК; частичное уменьшение объема следовой памяти также может свидетельствовать о нецелесообразности применения ПРАК для коррекции когнитивного статуса у данной когорты пациентов. Возможно, учитывая доказанную эффективность использования программ резонансноакустических колебаний в отношении широкого спектра заболеваний (Машков, Рыбкин, Цупко, 2017), в дальнейшем перспективы исследования могут ассоциироваться с изучением активирующего воздействия бинауральных биений на состояние ВПФ в процессе медицинской реабилитации.

Заключение (Conclusions). Использованная в качестве методического решения в настоящей работе процедуры кластерного анализа по методу К-средних представляет собой современный эргономичный вариант компактной подачи данных, позволяющий при анализе результатов применения стандартных (рутинных) нейропсихологических методик дискриминировать обследованную выборку в количественных градациях «низкого-среднего-высокого» уровня выражен- 
ности изучаемых характеристик с учетом многофакторной структуры эмпирических данных, что, в свою очередь, дает возможность решить вопрос об объективизации специфических локальных данных для обследованного контингента, без привязки к наличию/отсутствию единых нормативов, проследить динамику изменений в результате коррекционного воздействия, а также интегрировать полученные результаты в общенаучный контекст.

\section{Список литературы}

Астаева А.В. Психометрический подход к оценке результатов нейропсихологического обследования в детском возрасте // Сибирский психологический журнал. 2008. № 30. С. 107109.

Астаева А.В., Солянникова Н.С. Классификации современных зарубежных методик детской нейропсихологии в контексте психометрического подхода в психодиагностике детей // Вестник ЮУрГУ. Серия «Психология». 2018. № 1. С. 33-50.

Актуальные проблемы нейропсихологии детского возраста: Учебное пособие / Цветкова Л.С., Семенович А.В., Котягина С.Н., Гришина Е.Г., Гогберашвили Т.Ю. Под ред. Цветковой Л.С. 2-е изд., испр. М.: Издательство Московского психолого-социального института, 2006. 293 c.

Ахметзянова А.И. Практикум по клинической психологии: Учебно-методическое пособие. М.: Издательство «Школьная пресса», 2013. $78 \mathrm{c}$.

Ахутина Т.В., Каширская Е.В. Нейропсихологический анализ индивидуальных особенностей переработки лексической информации // Вопросы психологии. 2000. №3. С. 93-101.

Ахутина Т.В., Матвеева Е.Ю., Романова А.А. Применение луриевского принципа синдромного анализа в обработке данных нейропсихологического обследования детей с отклонениями в развитии // Вестник московского университета. Серия 14. Психология. 2012. №2. C. 84-95.

Ахутина Т.В., Меликян 3.А. Нейропсихологическое тестирование: обзор современных тенденций. К 110-летию со дня рождения А.Р. Лурия // Клиническая и специальная психология. 2012. Том 1. № 2. URL: https: // psyjournals.ru/psyclin/2012/n2/52599.shtml (дата обращения: 01.07.2020).
Балашова Е.Ю., Ковязина М.С. Нейропсихологическая диагностика. Классические стимульные материалы. М.: Генезис, 2016. 6-е издание. $70 \mathrm{c.}$

Беребин М.А., Астаева А.В. К вопросу о качественном и психометрическом подходах в современной нейропсихологической диагностике // Вестник ЮУрГУ. Серия «Психология». 2008. №32. С.19-28.

Блейхер В.М., Крук И.В. Патопсихологическая диагностика. Киев: Здоров'я, 1986. 280 с.

Вассерман Л.И., Дорофеева С.А., Меерсон Я.А. Методы нейропсихологической диагностики // СПб.: Стройлеспечать. 1997. 304 с.

Вассерман Л.И., Щелкова О.Ю. Медицинская психодиагностика: Теория, практика и обучение. СПб.: Филологический факультет СПбГУ; М.: Издательский центр «Академия», 2003. $756 \mathrm{c}$.

Глозман Ж.М. Количественная оценка данных нейропсихологического исследования. М.: Центр лечебной педагогики, 1999. 160 с.

Глозман Ж.М. Нейропсихологическое обследование: качественная и количественная оценка данных. М.: Смысл, 2012. 264 с.

Глозман Ж.М. Нейропсихология детского возраста: Учебное пособие для студ. высш. учеб. заведений. М.: Издательский центр «Академия», 2009. $272 \mathrm{c}$.

Гришина Д.А. Нейропсихологическая диагностика и лечение пациентов с деменцией // Медицинский совет. 2018. №18. С. 16-22. URL: https://cyberleninka.ru/article/n/neyropsihologiches kaya-diagnostika-i-lechenie-patsientov-sdementsiey (дата обращения: 01.07.2020).

Карвасарский Б.Д. Клиническая психология: Учебник. СПб: Питер, 2004. 960 с.

Крамер Д. Математическая обработка данных в социальных науках: современные методы: Учебное пособие для студ. высших учеб.заведений / Дункан Крамер; пер. с англ. И.В. Тимофеева, Я.И. Киселевой; науч. ред. О.В. Митина. М.: Издательский центр «Академия», 2007. $288 \mathrm{c}$.

Логвинова И.В. Методы нейропсихологического обследования детей в отечественной психологии (аналитический обзор) // Новосибирск: Психология и педагогика: методика и проблемы практического применения. 2010. №16-1. С. 211-220.

Лурия А.Р. Высшие корковые функции и их нарушения при локальных поражениях головного мозга. М.: Изд-во МГУ, 1962. 431 с. 
Машков О.А., Рыбкин Е.А., Цупко И.В. Восстановление психосоматического состояния человека методом воздействия программами резонансно-акустических колебаний (ПРАК). Пособие для врачей и пользователей. М., 2017. $30 \mathrm{c}$.

Менделевич В.Д. Клиническая и медицинская психология: Учебное пособие. / В.Д. Менделевич. 6-е изд. М.: МЕДпресс-информ, 2008. $432 \mathrm{c}$.

Методика организации психокоррекционных мероприятий в процессе медицинской реабилитации и восстановительного лечения (Методические рекомендации). / Кукшина А.А., Котельникова А.В., Турова Е.А., Рассулова М.А. M., 2017. 8 c.

Микадзе Ю.В. Нейропсихология детского возраста: Учебное пособие. СПб.: Питер, 2008. $288 \mathrm{c}$.

Микадзе Ю.В., Титкова И.И., Ширшов A.B., Гуща А.О. Когнитивные функции у пациентов с болезнью Паркинсона: динамика изменений после глубокой электростимуляции структур паллидарного комплекса // Анналы клинической и экспериментальной неврологии. 2013. № 3. С. 4-8.

Наследов А.Д. Математические методы психологического исследования. Анализ и интерпретация данных: учебное пособие для студентов высших учебных заведений, обучающихся по направлению и по специальности психологии. 3-е изд., стер. Санкт-Петербург: Речь, 2007. 389 c.

Петри А., Сэбин А. Наглядная медицинская статистика: Учебное пособие для вузов / пер. с англ. под ред. В.П. Леонова. 3-е изд., перераб. и доп. Москва: ГЭОТАР-Медиа, 2015. $214 \mathrm{c}$.

Рассказова Е.И., Ковязина М.С., Варако Н.А. Применение скрининговых шкал в нейропсихологической реабилитации: возможности, требования и ограничения // Вестник ЮУрГУ. Серия «Психология». 2016. № 3. С. 5-15.

Рубиштейн С.Я. Экспериментальные методики патопсихологии. М.: ЗАО Изд-во ЭКСМО-Пресс, 1999. 448 с.

Султанова А.С., Иванова И.А. К проблеме нормативных показателей в патопсихологической диагностике // Клиническая и специальная психология. 2017. Том 6. № 2. С.83-96.

Харисова Р.Р., Чебакова Ю.В. Практикум по патопсихологической диагностике: Учебное пособие / Под ред. Ю.В. Чебаковой. М.: Языки Народов Мира: ТЕЗАУРУС, 2018. 330 с.
Шумов Д.Е., Арсеньев Г.Н., Свешников Д.С., Дорохов В.Б. Сравнительный анализ влияния бинауральных биений и сходных видов звуковой стимуляции на процесс засыпания: короткое сообщение // Вестник Московского университета. Серия 16. Биология. 2017. №1 (72). C. 39-43.

Assessing Cognitive Function / Foreman M.D., Fletcher K., Mion L.C., Simon L. // Geriatric Nursing. 1996. №17. P. 228-233.

Butler M., Retzlaff P., Vanderploeg R. Neuropsychological test usage. Professional Psychology: Research and Practice, 1991. Vol. 22, №. 6. P. 510-512.

Byrne B. Adaptation of assessment scales in cross-national research: issues, guidelines and caveats // International Perspectives in Psychology: Research, Practice, Consultation. 2016. № 5(1). P. 51-65.

Folstein M.F., Folstein S.E., McHugh P.R. Mini-mental state. A practical method for grading the cognitive state of patients for the clinician // Journal of Psychiatric Research. 1975. № 12(3). P. 189-198.

Golden C.J. Manual for the Luria-Nebraska neuropsychological battery.Children Revision. Los Angeles: Western Psychological Services, 1981. $205 \mathrm{p}$.

Golden C., Espe-Pfeiffer P., WacheslerFelder J. Neuropsychological Interpretation of Objective Psychological Tests // Florida: Kluwer Academic Publishers, 2002. 241 p.

Lang T.A., Secic M. How to Report Statistics in Medicine: Annotated Guidelines for Authors, Editors, and Reviewers, 2nd edition. Philadelphia: American College of Physicians, 2006. 490 p.

Lerner A.J. Cognitive screening instruments.A practical approach. London: Springer, 2013. 247 p.

Nasreddine Z.S., Phillips N.A., Bédirian V., Charbonneau S., Whitehead V., Collin I., Cummings J.L., Chertkow H. The Montreal Cognitive Assessment, MoCA: a brief screening tool for mild cognitive impairment // J Am Geriatr Soc. 2005. № 53(4). P. 695-699.

Neuropsychological Assesment / Lezak M.D., Howieson D.B., Bigler E.D., Tranel D. 5th ed. New York: Oxford University Press, 2012. $1200 \mathrm{p}$.

Ramachandran K.M. and Tsokos C.P. Mathematical statistics with applications / Kandethology M. Ramachandran, Chris P.Tsokos. Florida: Academic Press, 2009. 849 p. 


\section{References}

Astaeva, A.V. (2008), "A psychometric approach to assessment issues in child neuropsychology", Siberian Journal of Psychology, 30, 107-109. (In Russian).

Astaeva, A.V. and Solyannikova, N.S. (2018), "Classification of modern foreign methods of pediatric neuropsychology in the context of the psychometric approach in the psychodiagnosis of children", Bulletin of the South Ural State University, Series "Psychology", 1, 33-50. (In Russian).

Tsvetkova, L.S., Semenovich, A.V., Kotyagina, S.N., Grishina, E.G. and Gogberashvili T.Yu. (2006), Aktual'nye problemy neiropsikhologii detskogo vozrasta: Uchebnoe posobie [Current problems of child neuropsychology: A Study Guide], in Tsvetkova, L.S. (ed.), 2 nd ed., Izdatel'stvo Moskovskogo psikhologo-sotsial'nogo institute, Moscow, Russia.

Akhmetzyanova, A.I. (2013), Praktikum po klinicheskoi psikhologii: Uchebno-metodicheskoe posobie [A manual in clinical psychology: Educational and methodological guide], Izdatel'stvo «Shkol'naya pressa», Moscow, Russia.

Akhutina, T.V. and Kashirskaya, E.V. (2000), "Neuropsychological analysis of individual characteristics of processing lexical information", Questions of Psychology, 3, 93-101. (In Russian).

Akhutina, T.V., Matveeva, E.Yu. and Romanova, A.A. (2012), "Application of the Luria's principle of the syndrome analysis in processing of neuropsychological assessment data of children with developmental disorders", University Psychology Bulletin, Series 14, Psychology, 2, 84-95. (In Russian).

Akhutina, T.V. and Melikyan, Z.A. (2012), "Neuropsychological Assessment: an overview of modern tendencies (dedicated to 110-th anniversary of A.R. Luria)", Clinical Psychology and Special Education, 1 (2), available at: https://psyjournals.ru/psyclin/2012/n2/52599.shtml (Accessed 15 April 2019). (In Russian).

Balashova, E.Yu. and Kovyazina, M.S. (2016), Neiropsikhologicheskaya diagnostika. Klassicheskie stimul'nye materialy [Neuropsychological diagnosis.Classical stimulus materials], Genezis, Moscow, Russia.

Berebin, M.A. and Astaeva, A.V. (2008), "On the qualitative and psychometric approaches in contemporary neuropsychological assessment", Bulletin of the South Ural State University, Series "Psychology", 32, 19-28. (In Russian).
Bleikher, V.M. and Kruk, I.V. (1986), Patopsikhologicheskaya diagnostika [Pathopsychological diagnostics], Zdorov'ya, Kyiv, Ukraine.

Vasserman, L.I., Dorofeeva, S.A. and Meerson, Ya.A. (1997), Metody neiropsikhologicheskoi diagnostiki [Methods of neuropsychological diagnostics], Stroilespechat', Saint Petersburg, Russia.

Vasserman, L.I. and Shchelkova, O.Yu. (2003), Meditsinskaya psikhodiagnostika: Teoriya, praktika i obuchenie [Medical psychodiagnostics: Theory, practice and education], Izdatel'skii tsentr Akademiya, Moscow, Russia.

Glozman, Zh.M. (1999), Kolichestvennaya otsenka dannykh neiropsikhologicheskogo issledovaniya [Quantitative assessment of neuropsychological research data], Tsentr lechebnoi pedagogiki, Moscow, Russia.

Glozman, Zh.M. (2012), Neiropsikhologicheskoe obsledovanie: kachestvennaya $i$ kolichestvennaya otsenka dannykh [Neuropsychological research: qualitative and qualitative data assessment], Smysl, Moscow, Russia.

Glozman, Zh.M. (2009), Neiropsikhologiya detskogo vozrasta: Uchebnoe posobie dlya stud. vyssh. ucheb. zavedenii [Childhood neuropsychology: A Study Guide for students of higher educational institutions], Izdatel'skii tsentr Akademiya, Moscow, Russia.

Grishina, D.A. (2018), "Neuropsychological testing in the diagnosis and management of patients with dementia", Medical Council, 18, 16-22, available at: https: // cyberleninka.ru/article/n/neyropsihologicheskaya-diagnostikai-lechenie-patsientov-s-dementsiey (Accessed 01 July 2020).

Karvasarskii, B.D. (2004), Klinicheskaya psikhologiya: Uchebnik [Clinical psychology: Textbook], Piter, Saint Petersburg, Russia.

Kramer, D. (2007), Matematicheskaya obrabotka dannykh $v$ sotsial'nykh naukakh: sovremennye metody: Ucheb. posobie dlya stud. vysshikh ucheb. zavedenii [Mathematical data processing in social Sciences: modern methods: A Study Guide for students of higher educational institutions], Translated by Timofeeva, I.V., Kiseleva, Ya.I., in Mitina, O.V. (ed.), Izdatel'skii tsentr Akademiya, Moscow, Russia.

Logvinova, I.V. (2010), "Methods of neuropsychological research of children in Russian psychology (analytical review)", Psikhologiya i pedagogika: metodika $i$ problemy prakticheskogo primeneniya, 16-1, 211-220. (In Russian).

Luriya, A.R. (1962), Vysshie korkovye funktsii i ikh narusheniya pri lokal'nykh porazheni- 
yakh golovnogo mozga [Human Higher Cortical Functions and Their Impairments in Case of Local Brain Lesions], Izd-vo MGU, Moscow, Russia.

Mashkov, O.A., Rybkin, E.A. and Tsupko, I.V. (2017), Vosstanovlenie psikhosomaticheskogo sostoyaniya cheloveka metodom vozdeistviya programmami rezonansno-akusticheskikh kolebanii (PRAK).Posobie dlya vrachei i pol'zovatelei [Restoration of the psychosomatic state of a person by the method of creating programs of resonant acoustic vibrations (practice). Manual for doctors and users], Moscow, Russia.

Mendelevich, V.D. (2008), Klinicheskaya $i$ meditsinskaya psikhologiya: Uchebnoe posobie [Clinical and medical psychology: A Study Guide], 6 th ed., MEDpress-inform, Moscow, Russia.

Mikadze Yu.V., Titkova I.I., Shirshov A.V. and Gushcha A.O. (2013), "Changes in cognitive functions after deep-brain stimulation of the globus pallidus in patients with Parkinson disease". Annals of Clinical and Experimental Neurology, 3, 4-8. (In Russian).

Kukshina, A.A., Kotel'nikova, A.V., Turova, E.A. and Rassulova, M.A. (2017), Metodika organizatsii psikhokorrektsionnykh meropriyatii $v$ protsesse meditsinskoi reabilitatsii $i$ vosstanovitel'nogo lecheniya (Metodicheskie rekomendatsii) [The methods of organization of psychocorrective activities in the process of medical rehabilitation and restorative treatment (Guidelines)], Moscow, Russia.

Mikadze, Yu.V. (2008), Neiropsikhologiya detskogo vozrasta: Uchebnoe posobie [Child neuropsychology: A Study Guide], Piter, Saint Petersburg, Russia.

Nasledov, A.D. (2015), Matematicheskie metody psikhologicheskogo issledovaniya. Analiz $i$ interpretatsiya dannykh: uchebnoe posobie dlya studentov vysshikh uchebnykh zavedenii, obuchayushchikhsya po napravleniyu $i$ po spetsial'nosti psikhologii [Mathematical methods of psychological research. Data analysis and interpretation: a study guide for students of higher educational institutions studying in the field and specialty of psychology], 3 d ed., Rech', Saint Peterburg, Russia.

Petri, A. and Sebin, K. (2015), Naglyadnaya meditsinskaya statistika: Uchebnoe posobie dlya vuzov [Visual medical statistics: A study guide for universities], in Leonov, V. P. (ed.), 3 d ed., GEOTAR-Media, Moscow, Russia.

Rasskazova, E.I., Kovyazina, M.S. and Varako, N.A. (2016), "Screening scales in neuropsycho- logical rehabilitation: opportunities, requirements and limitations", Bulletin of the South Ural State University. Series "Psychology”, 3, 5-15. (In Russian).

Rubishtein, S.Ya. (1999), Eksperimental'nye metodiki patopsikhologii [Experimental methods of pathopsychology], ZAO Izd-vo EKSMO-Press, Moscow, Russia.

Shumov, D.E., Arsen'ev, G.N., Sveshnikov, D.S. and Dorokhov, V.B. (2017), "Comparative analysis of the effect of the stimulation with binaural beat and similar kinds of sound on falling asleep process: a brief note", Moscow University Psychology Bulletin. Series 16, Biology, 72 (1), 39-43. (In Russian).

Sultanova, A.S. and Ivanova, I.A. (2017), "To the Problem of Normative Data in Pathopsychological Diagnostics", Clinical Psychology and Special Education, 6 (2), 83-96. (In Russian).

Kharisova, R.R. and Chebakova, Yu.V. (2018), Praktikum po patopsikhologicheskoi diagnostike: Uchebnoe posobie [A practical course on pathopsychological diagnostics: A study guide], in Chebakova Yu. V. (ed.), Yazyki Narodov Mira: TEZAURUS, Moscow, Russia.

Foreman, M.D., Fletcher, K., Mion, L.C. and Simon, L. (1996), "Assessing Cognitive Function"Geriatric Nursing, № 17, 228-233. (In Russian).

Butler, M., Retzlaff, P. and Vanderploeg R. (1991), "Neuropsychological test usage" Professional Psychology: Research and Practice, 22 (6), 510-512.

Byrne, B. (2016), “Adaptation of assessment scales in cross-national research: issues, guidelines and caveats" International Perspectives in Psychology: Research, Practice, Consultation, № 5 (1), 51-65.

Folstein, M.F., Folstein, S.E. and McHugh, P.R. (1975), "Mini-mental state.A practical method for grading the cognitive state of patients for the clinician" Journal of Psychiatric Research, № 12 (3), 189-198.

Golden, C.J. (1981), Manual for the LuriaNebraska neuropsychological battery. Children Revision, Western Psychological Services, Los Angeles, USA.

Golden, C., Espe-Pfeiffer, P. and WacheslerFelder J. (2002), Neuropsychological Interpretation of Objective Psychological Tests, Florida, USA.

Lang, T.A. and Secic M. (2006), How to Report Statistics in Medicine: Annotated Guidelines 
for Authors, Editors, and Reviewers, 2 nd ed, American College of Physicians, Philadelphia, USA.

Lerner, A.J. (2013), Cognitive screening instruments. A practical approach, Springer, London, UK.

Lezak, M.D., Howieson, D.B., Bigler, E.D. and Tranel D. (2012) Neuropsychological Assesment, 5th ed, Oxford University Press, New York, USA.

Ramachandran, K.M. and Tsokos, C.P. (2009), Mathematical statistics with applications, Academic Press, Florida, USA.

Nasreddine, Z.S., Phillips, N.A., Bédirian, V., Charbonneau, S., Whitehead, V., Collin, I., Cummings, J. L. and Chertkow, H. (2005), "The Montreal Cognitive Assessment, MoCA: a brief screening tool for mild cognitive impairment", $J$ Am Geriatr Soc, 53(4), 695-699.

Информация о конфликте интересов: авторы не имеют конфликта интересов для декларации.

Conflicts of Interest: the authors have no conflict of interests to declare.

\section{Данные авторов:}

Тихонова Анастасия Сергеевна, медицинский психолог, Московский научно-практический центр медицинской реабилитации, восстановительной и спортивной медицины Департамента здравоохранения города Москвы. ORCID: 00000001-7693-9397.

Котельникова Анастасия Владимировна,
кандидат психологических старший научный сотрудник отдела медицинской реабилитации, Московский научнопрактический центр медицинской реабилитации, восстановительной и спортивной медицины Департамента здравоохранения города Москвы. ORCID: 0000-0003-1584-4815.

Кукшина Анастасия Алексеевна, доктор медицинских наук, ведущий научный сотрудник отдела медицинской реабилитации, Московский научно-практический центр медицинской реабилитации, восстановительной и спортивной медицины Департамента здравоохранения города Москвы. ORCID: 0000-0002-2290-3687.

\section{About the authors:}

Anastasiya S. Tikhonova, Medical Psychologist, Moscow Centre for Research and Practice in Medical Rehabilitation, Restorative and Sports Medicine, Moscow Healthcare Department. ORCID: 00000001-7693-9397.

Anastasiya V. Kotel'nikova, Candidate of Psychological Science, Associate Professor, Senior Research Assistant in Medical Rehabilitation, Moscow Centre for Research and Practice in Medical Rehabilitation, Restorative and Sports Medicine, Moscow Healthcare Department. ORCID: 0000-00031584-4815.

Anastasiya A. Kukshina, Doctor of Medicine, Leading Research Assistant, Moscow Centre for Research and Practice in Medical Rehabilitation, Restorative and Sports Medicine of Moscow Healthcare Department. ORCID: 0000-0002-22903687. 